\title{
Roles of matrix metalloproteinase-26 in the growth, invasion and angiogenesis of breast cancer
}

\author{
HONGFA YANG ${ }^{1,2}$, YANG WANG ${ }^{1}$, YILEI LI ${ }^{1}$, LIHONG ZHANG ${ }^{1}$, YIPING DENG ${ }^{1}$, \\ DONGXUE QI ${ }^{1}$, YULIN LI ${ }^{1}$ and WEI LI ${ }^{1}$ \\ ${ }^{1}$ The Key Laboratory of Pathobiology, Ministry of Education, Norman Bethune College of Medicine; \\ ${ }^{2}$ Department of Neurosurgery, The First Clinical Hospital, Jilin University, Changchun, Jilin 130021, P.R. China
}

Received April 5, 2012; Accepted July 17, 2012

DOI: $10.3892 / \mathrm{ol} .2012 .833$

\begin{abstract}
Matrix metalloproteinase-26 (MMP-26) is a novel member of the MMP family and plays a significant role in the progression of estrogen-dependent malignancies. The present study aimed to investigate the roles of MMP-26 in the growth, invasion and angiogenesis of breast cancer. pcDNA3.1(+)-neo expression plasmids carrying the proMMP-26 coding sequence were used to transfect a breast cancer cell line (MCF-7 cells). The mRNA and protein expression of MMP-26 was determined by RT-PCR, immunofluorescence analysis and flow cytometry. The morphology of transfected cells was observed under an electron microscope. An adherence and spreading assay, Boyden chamber assay, in vivo tumorigenicity assay and in vivo angiogenesis were further modeled to elucidate the roles of MMP-26 in the invasion and angiogenesis of breast cancer. Using electron microscopy, the MMP-26-transfected cells demonstrated increased atypia, including unusual mitotic figures, glucogen pools and special lysosomes in the cytoplasm. The adherence and spreading ability of MMP-26-transfected cells were increased significantly compared with cells in the control group. The Boyden chamber assay demonstrated that the migration and invasion ability of MMP-26-transfected cells was dramatically accelerated compared with the control group, but markedly reduced in the presence of anti-MMP-26 antibody. MMP-26 also increased the malignant phenotype in vivo. The number of vessel branches and the total length of vessels induced by MMP-26-transfected cells were significantly increased compared to those induced by non-transfected cells. The plasmid carrying the proMMP-26 gene was successfully transfected into breast cancer cells. Our results demonstrate that MMP-26 overexpression promotes the growth and invasion of breast cancer cells and induces angiogenesis.
\end{abstract}

Correspondence to: Professor Wei Li, The Key Laboratory of Pathobiology, Ministry of Education, Norman Bethune College of Medicine, 126 Xinmin Street, Jilin University, Changchun, Jilin 130021, P.R. China

Email: li1663@yeah.net

Key words: matrix metalloproteinase-26, breast cancer, invasion, transfection, angiogenesis

\section{Introduction}

Currently, the acceptable predictors of cancers include the tumor type and malignant potential of the respective tumor, including its proliferation, invasion and angiogenesis properties. An increasing number of studies indicate that matrix metalloproteinases (MMPs) contribute to the malignant phenotype, and that the evaluation of MMP expression may be helpful for the assessment of patients' prognosis (1-5). MMPs are a family of zinc-dependent endopeptidases with multiple functions, including proteolytic activity. Studies have demonstrated that MMPs play essential roles in numerous pathological processes such as tissue remodeling, wound healing, angiogenesis, apoptosis and tumor progression. MMPs not only degrade almost all the extracellular matrix (ECM) components, thereby promoting cancer invasion and metastasis (6), but also regulate cellular adhesion (7) and promote cancer angiogenesis (8).

MMP-26, a novel member of the MMP family, was first cloned in 2000 (9). MMP-26 has a variety of properties that distinguish it from other MMPs. It lacks a hinge region. The conservative PRCGXXD cysteine switch is replaced by PHCGVPD in MMP-26, which is the basis of its unorthodox activation and distinct functions (10). In order to investigate the roles of MMP-26 in the growth, invasion and angiogenesis of breast cancer, we prepared the recombinant plasmid pcDNA3.1(+)-neo carrying the proMMP-26 coding sequence, and transfected it into breast cancer MCF-7 cells. MMP-26 expression was measured by RT-PCR, immunofluorescence assay and flow cytometry. The observations of in vitro and in vivo growth and the invasive potential of MMP-26-transfected cells indicated that MMP-26 overexpression was closely correlated with the malignant phenotype, increased invasion ability and enhanced angiogenesis in the cancers. Thus, we speculated that the evaluation of MMP-26 expression may have clinical implications in predicting the prognosis of breast cancer.

\section{Materials and methods}

Cell line and cell culture. The human breast carcinoma cell line (MCF-7 cells) was purchased from ATCC and grown in H-Dulbecco's modified Eagle's medium (DMEM; Gibco, Invitrogen Life Technologies, Carlsbad, CA, USA) containing 
$10 \%$ fetal bovine serum (FBS; Sigma, St. Louis, MO, USA) at $37^{\circ} \mathrm{C}$. The ethics approval was given by the medical ethics committee of Basic Medical College, Jilin University, Jilin, China.

DNA transfection and clonal selection. pcDNA3.1(+)-neo expression plasmid carrying the proMMP-26 coding sequence (provided by Dr Alex Strongin; Burnham Institute, La Jolla, CA, USA) (8), was used to transfect MCF-7 cells using SuperFect (Qiagen, Hilden, Germany) following the manufacturer's instructions. An 800- $\mu \mathrm{g} / \mathrm{ml}$ concentration of G418 (Sigma) was used to select the MMP-26 stable transfectants. Single clones with G418 resistance were selected and expanded for further analysis. MMP-26 expression was determined in MCF-7 cells transfected with the pcDNA3.1 plasmid and in non-transfected MCF-7 cells, used as controls, by the methods described below.

Reverse transcriptase-polymerase chain reaction (RT-PCR). Total RNA was extracted using TRIzol reagent (Invitrogen Life Technologies) according to the manufacturer's instructions. cDNA was synthesized using AMV reverse transcriptase (Takara Bio Inc., Tokyo, Japan), oligo dT and $2 \mu \mathrm{g}$ total RNA. The reaction was carried out at $48^{\circ} \mathrm{C}$ for $30 \mathrm{~min}, 99^{\circ} \mathrm{C}$ for $5 \mathrm{~min}$ and $5^{\circ} \mathrm{C}$ for $5 \mathrm{~min}$. In the PCR assay, cDNA was amplified by 30 cycles of reactions (denaturing at $94^{\circ} \mathrm{C}$ for $30 \mathrm{sec}$, annealing at $55^{\circ} \mathrm{C}$ for $30 \mathrm{sec}$ and extension at $72^{\circ} \mathrm{C}$ for $1 \mathrm{~min}$ ) using primers for MMP-26 (forward, 5'-TGACATGCAGATGCATGCTCTGC-3'; and reverse, 5'-CTAGGGTCGTGATACCAGTAAGTG-3') according to a method previously described (9). The anticipated size of the PCR products was $500 \mathrm{bp}$. $\beta$-actin was amplified (forward, 5'-TGGAATCCTGTGGCATCCATGAAAC-3'; and reverse, 5'-TAAAACGCAGCTCAGTAACAGTCCG-3') and the expected size of $\beta$-actin was $360 \mathrm{bp}$.

Immunofluorescence assay. The cultured cells were fixed for $30 \mathrm{~min}$ in $4 \%$ paraformaldehyde, and permeabilized in $0.1 \%$ Triton X-100 (Sigma) for $10 \mathrm{~min}$. The endogenous peroxidase was inactivated with hydrogen peroxide ( $0.3 \%$ in methanol) followed by washing in phosphate-buffered saline (PBS). The cells were incubated with blocking serum for $30 \mathrm{~min}$ at room temperature and then with anti-human MMP-26 polyclonal antibody (a gift from Burnham Institute) (8) for $1 \mathrm{~h}$ at $37^{\circ} \mathrm{C}$ followed by washing in PBS. FITC-conjugated anti-rabbit antibody (Sigma) was used to treat the cells for $30 \mathrm{~min}$ followed by washing for $30 \mathrm{~min}$ at room temperature. The primary antibody was replaced with PBS serving as a negative control.

Flow cytometry. The MMP-26-transfected MCF-7 cells were harvested and fixed in $4 \%$ paraformaldehyde for $60 \mathrm{~min}$ at $4^{\circ} \mathrm{C}$. After washing in PBS, the cells were treated with $0.1 \%$ Triton X-100 for 10 min and washed in PBS. Anti-human MMP-26 polyclonal antibody was used to treat the cells for $40 \mathrm{~min}$ at $4^{\circ} \mathrm{C}$ followed by washing in PBS. FITC-conjugated anti-rabbit antibody was applied to treat these cells for $40 \mathrm{~min}$ at $4^{\circ} \mathrm{C}$ followed by washing. The cells were then re-suspended in $500 \mu \mathrm{l}$ PBS and subjected to flow cytometry.

Spreading of tumor cells on Matrigel. A single cell suspension in serum-free medium was firstly prepared. Cells $\left(1 \times 10^{4}\right)$
A

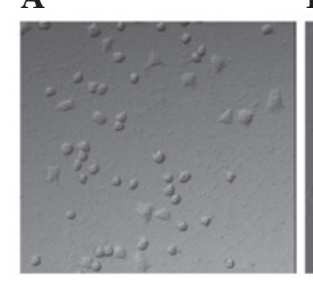

B

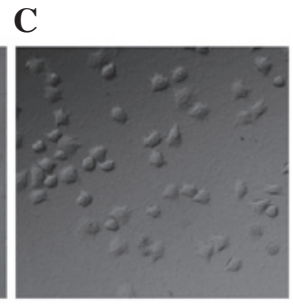

Figure 1. Spreading of MMP-26-transfected cells. (A) MCF-7 group; (B) pcDNA3.1 group; (C) MMP-26 group. The shape of cells became polygonal, resembling pseudopods (magnification, $\mathrm{x} 200$ ).

were seeded into 96-well plates precoated with Matrigel $^{\mathrm{TM}}$ (BD Biosciences, Franklin Lakes, NJ, USA). The cells were grown for $1.5 \mathrm{~h}$ and washed with PBS. The morphology of spreading cells was observed under the microscope.

Boyden chamber assay. Boyden chambers (Falcon; BD Biosciences) were precoated with Matrigel (60 $\mu \mathrm{l} / \mathrm{well})$ and incubated for $30 \mathrm{~min}$ at $37^{\circ} \mathrm{C}$. The Matrigel precoated chamber was supplemented with anti-MMP-26 polyclonal antibody $(100 \mu \mathrm{g} / \mathrm{ml})$ in the MMP-26 transfected group. Cells in $200 \mu \mathrm{l}$ serum-free H-DMEM were stained with rhodamine (Invitrogen Life Technologies) and seeded into the upper chambers. The lower chambers were filled with NIH3T3 culture supernatant to create a chemotactic gradient. Incubation was performed at $37^{\circ} \mathrm{C}$ with $5 \% \mathrm{CO}_{2}$. In the migration assay, the distances that cells migrated on the Matrigel were measured under a laser confocal microscope after $2 \mathrm{~h}$. In the invasion assay, the cells on the upper surface with the Matrigel were removed by wiping the surface firmly with a cotton swab after $4 \mathrm{~h}$ of incubation. The filters were photographed under a laser confocal microscope (Olympus, Tokyo, Japan). The number of cells penetrating the filter was counted under the microscope at a magnification of $x 100$. Ten visual fields were counted on each filter. The results are expressed as the mean \pm SD.

In vivo nude mice bearing breast cancer model. Cells $\left(1 \times 10^{5}\right)$ were harvested and inoculated into the groin region of 6-8-week-old female nude mice (nu/nu; Lianhelihua Company, Beijing, China). The mice were bred in the Experimental Animal Center of Jilin University (Changchun, Jilin, China). Twenty days later, the mice were sacrificed and the tumors were collected. The volumes of solid tumors were estimated by measuring the long and short diameter of the tumor. Tumor tissues were fixed in 10\% formalin, paraffin-embedded and cut into 5- $\mu \mathrm{m}$ sections. Hematoxylin and eosin staining was then performed. The morphologic characteristics of the tumors were observed under a light microscope.

Tumor cell-induced angiogenesis model. Cells $\left(1 \times 10^{5}\right)$ were harvested and inoculated subcutaneously into the backs of nude mice. The mice were sacrificed after 4 days. The overlying skin was collected. The injection site was photographed under the dissecting microscope (magnification, $\mathrm{x} 7$ ). Ten visual fields of each nude mouse were employed and the number of blood vessels was counted. The results are expressed as the mean \pm SD of vessel numbers per visual field. 
Table I. Angiogenesis in tumors following allogeneic cancer cell inoculation.

\begin{tabular}{lccr}
\hline Groups of cells & No. of branches & $\begin{array}{c}\text { Total length of } \\
\text { vascular branches }(\mathrm{cm})\end{array}$ & P-value \\
\hline Non-transfected MCF-7 & $15.3 \pm 2.50$ & $17.02 \pm 10.07$ & $<0.05^{\mathrm{a}}$ \\
pcDNA3.1-transfected & $24.4 \pm 10.0$ & $31.68 \pm 3.78$ & \\
MMP-26-transfected & $47.0 \pm 13.8$ & $47.70 \pm 8.75$ & \\
\hline
\end{tabular}

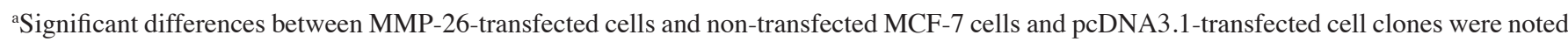
$(\mathrm{P}<0.05)$.

A

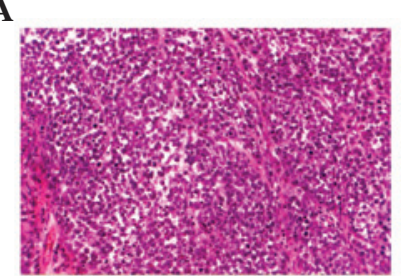

E

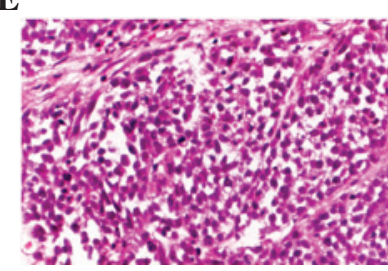

B

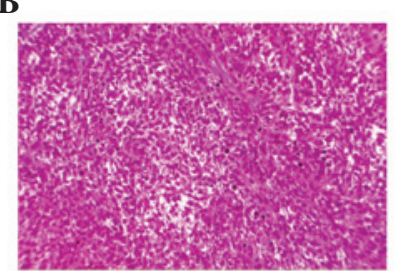

F

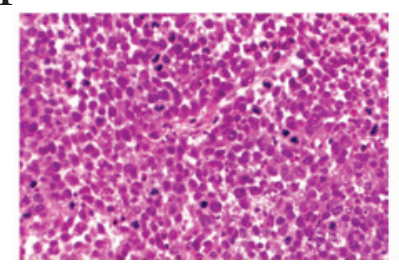

C

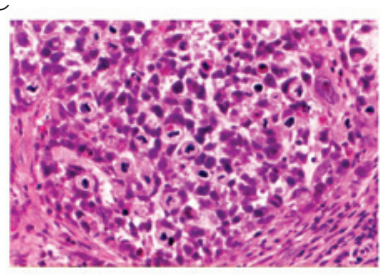

D

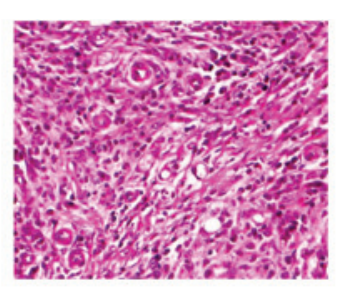

G

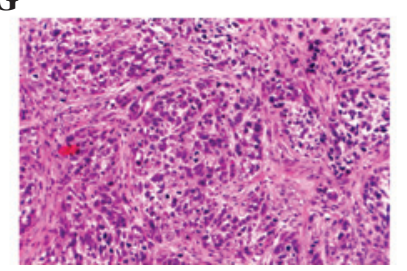

Figure 2. Xenogeneic breast cancer following MMP-26 transfection. (A, B, C and D) Magnification, x200; (E, F and G) Magnification, x400. (A and E) MCF-7 group; (B and F) pcDNA3.1 group; (C) MMP-26-transfected group; (D) MMP-26 transfected group, the newly generated blood vessels dramatically increased in stroma; (G) MMP-26-transfected cells were larger in size with increased mitotic figures.

\section{Results}

Expression of MMP-26 mRNA in MMP-26-transfected cells. MCF-7 cells were transfected with pcDNA3.1(+)-neo plasmid carrying the proMMP-26 coding sequence. Following G418 resistance screening, neomycin-resistant clones were selected and the expression of MMP-26 mRNA was determined by RT-PCR. Results showed that the expression level of MMP-26 mRNA was significantly increased in MMP-26 transfected cells compared to non-transfected MCF-7 cells and pcDNA3.1(+) vector-transfected MCF-7 cells.

Immunofluorescence assay of MMP-26 expression. MMP-26 polyclonal antibody was used to determine MMP-26 expression in MMP-26-transfected cells and cells in controls. Results showed that the protein expression of MMP-26 was significantly increased in the MMP-26-transfected cells compared with that in control groups. The immunofluorescence assay demonstrated high expression of MMP-26 in the cytoplasm of MMP-26-transfected cells compared with weak expression in MCF-7 cells and pcDNA3.1(+) vector-transfected cells.

Protein expression of MMP-26 by flow cytometry. MMP-26 protein expression was also determined by flow cytometry. The peak of MMP-26 in the MMP-26-transfected cells demonstrated a rightward shift and the average intensity was doubled compared to the non-transfected MCF-7 cells and pcDNA3.1-transfected cell clones.

Morphologic changes of MMP-26-transfected MCF-7 cells. Following transfection with the pcDNA3.1(+)-neo expression plasmid carrying the proMMP-26 coding sequence, the MCF-7 cells showed evident morphologic changes compared to the control groups. The cells appeared larger and more pleomorphic with abnormal nuclei. The ultrastructure of transfected cells were as follows: the number of mitotic cells increased, pathological karyokinesis was noted and myelin-like bodies appeared in the cytoplasm of several cells. These features indicate high proliferation and a high degree of malignancy.

Spreading of MMP-26-transfected cells. The MMP-26transfected cells and those in the control group were seeded into 96-well plates precoated with Matrigel and were grown in serum-free $\mathrm{H}$-DMEM at $37^{\circ} \mathrm{C}$ for $1.5 \mathrm{~h}$. The spreading ability of MMP-26 transfected cells increased considerably compared to the control groups. The shape of cells became polygonal and more pseudopods were observed (Fig. 1).

Migration and invasion of MMP-26-transfected cells. To detect the migration of the transfected cells in the Boyden chambers, a laser confocal microscope was employed to 
observe cells $2 \mathrm{~h}$ after incubation in the Boyden chamber precoated with Matrigel. Results showed the migration ability of MMP-26-transfected cells was markedly higher compared with the control group. The migration ability of MMP-26-transfected cells was dramatically reduced in the presence of MMP-26 antibody. Four hours after incubation, the number of cells that invaded the filter was counted. The number of invasive cells in the MMP-26-transfected cells was significantly higher than in the control group $(\mathrm{P}<0.01)$. The number of invasive cells in the presence of MMP-26 antibody was significantly reduced $(\mathrm{P}<0.01)$.

Growth of MCF-7 cell-induced breast cancer in nude mice. MMP-26-transfected, pcDNA3.1-transfected and non-transfected MCF-7 cells were inoculated subcutaneously into nude mice and the tumor growth was detected in vivo. Solid tumors formed 7 days after inoculation. Twenty days later, the tumors were collected, and tumor size and weight were measured. Results showed that there was no significant difference in the tumor size among the three groups ( $>>0.05$; data not shown). Under a microscope (magnification, $\mathrm{x} 200$ ), the cancer nests in the mice inoculated with MMP-26-transfected cells were smaller than those in the controls, with more surrounding stroma (Fig. 2A, B and C). The angiogenesis in the tumors of nude mice treated with MMP-26-transfected cells (Fig. 2D) was dramatically increased compared with that in the controls.

At high magnification (x400), the parenchymal cells in the tumors of mice treated with MMP-26-transfected cells had larger size and were more pleomorphic than those in controls. There were more tumor giant cells (Fig. 2E) and more mitotic figures, including more atypical mitotic figures (Fig. 2F) than the controls (Fig. 2G). These findings indicate that the tumors of mice treated with MMP-26-transfected cells were highly malignant and had higher proliferation when compared with tumors of mice treated with cells in the control group.

MMP-26-transfected cells induced angiogenesis. MMP-26-transfected, pcDNA3.1-transfected and non-transfected MCF-7 cells were inoculated subcutaneously into nude mice and the angiogenesis of formed tumors was evaluated. The skin was removed 4 days later and the blood vessels were measured. Results showed that MMP-26-transfected cell-induced tumors had significantly increased angiogenesis as compared to the control group. The newly generated blood vessels were large and abundant in branches forming the vascular networks. Quantitative analysis of the newly generated blood vessels revealed that the number of vascular branches and the total length of these vessels in MMP-26-transfected cell induced tumors were significantly different from those in control cell-induced tumors $(\mathrm{P}<0.01$; Table I).

\section{Discussion}

The invasion and metastasis of malignant tumors is a complex and multi-stage process associated with the enhancement of proteolytic activity and the degradation of the ECM. There are four main types of proteolytic enzymes taking part in the degradation of ECM, among which MMPs are the largest family with the most complicated functions and high proteolytic activity. MMPs are involved in the metastasis and invasion of cancers by degrading the ECM, regulating cell adherence and promoting angiogenesis (1-5).

MMP-26 is a novel member of the MMP family. Numerous studies have been conducted to investigate the prokaryotic expression, spatial structure, in vitro activation and the substrate cleavage specificity of MMP-26 (9-13). However, the functions of MMP-26 in cancer progression and their clinical significance are still poorly understood. Herein, pcDNA3.1 vector carrying the full-length gene of MMP-26 was transfected into MCF-7 cells, a human breast cancer cell line, and the roles of MMP-26 in the malignant phenotype of these cells were evaluated.

In the present study, the cellular atypia of MCF-7 cells increased significantly once they were transfected with the MMP-26 gene in vivo and in vitro. In vitro, the MMP-26-transfected cells were larger and more pleomorphic with more tumor giant cells compared with the non-transfected cells and pcDNA3.1-transfected cells. In vivo, MMP-26-transfected cells had similar morphologic features to those in vitro. The parenchymal cells in the MMP-26-transfected cell-induced tumors appeared to be more pleomorphic with more tumor giant cells and more mitotic figures, including atypical mitotic figures. All of these findings suggest that the overexpression of MMP-26 turned the less malignant phenotype of MCF-7 cells into a highly malignant phenotype. The increased number of mitotic figures also suggests enhanced cell proliferation. The mechanism of MMP-26-induced promotion of cell proliferation has not been elucidated to date. Golubkov et al (14) hypothesized that MMPs acted as oncogenes promoting the malignant transformation of normal cells rather than just as enzymes supporting the growth of pre-existing cancers. To validate this hypothesis, normal 184B5 human mammary epithelial cells were transfected with MT1-MMP (184B5-MT1 cells). Results showed that 184B5-MT1 cells exhibited aneuploidy and were efficient in generating cancers in an orthotopic xenograft model in immunodeficient mice. They also found that the oncogenic functions of MT1-MMP were related to its proteolysis of pericentrin, one of the most notable scaffolding proteins of pericentriolar material surrounding the centrosome (15). These observations may be useful for further studies on the oncogenic mechanism of MMP-26.

Penetration of the basement membrane by breast cancer cells is a key step in which in situ cancer becomes infiltrating cancer. Thus, the migration and invasion of cancer cells are important indicators in evaluating the degree of malignancy. In the present study, MMP-26-transfected cells adhered to the Matrigel more rapidly and had increased pseudopodia or altered shapes (from round to polygonal) when compared with cells in the control group. In the migration and invasion assay, MMP-26-transfected cells had significantly increased migration and invasion through the filter as compared to the cells in the control group. However, the migration and invasion of MMP-26-transfected cells were effectively inhibited in the presence of MMP-26 antibody. These results suggest that MMP-26 promotes the adherence, migration and invasion of MCF-7 cells. Matrigel is an analog of the basement membrane and its components and structure are similar to to those of the basement membrane in vivo. Therefore, we speculate that MMP-26 may play a key role in the early invasion of breast 
cancer by promoting adhesion to the basement membrane and migration or invasion through it. By immunofluorescence microscopy, Zhao et al (16) detected MMP-26 expression in human breast ductal carcinoma in situ (DCIS), infiltrating ductal carcinoma (IDC), atypical intraductal hyperplasia and normal breast epithelia adjacent to ductal DISC and IDC. Their results revealed that MMP-26 expression in DCIS was significantly higher than in the other tissues. We postulate that the increased MMP-26 expression in DCIS may promote the infiltrating ability of the cancer cells, allowing them to eventually penetrate the basement membrane.

Cancer growth is also dependent on the angiogenesis within it. The blood vessels in cancers not only supply nutrition, but also provide potential pathways for hematogenous metastasis. A variety of factors produced by the cancer cells induce angiogenesis in cancers, including MMPs (3). In the present study, angiogenesis in MMP-26-transfected cell-induced cancers was significantly promoted and the blood vessels were larger in diameter and longer in total length than those in the MCF-7 and pcDNA3.1-transfected cell-induced cancers. In short, there were more newly generated capillaries in the stroma of MMP-26-transfected cell-induced cancers than in the control group. These findings demonstrate that MMP-26 is a potent inducer of angiogenesis in cancers.

As a novel member of MMPs, the function of MMP-26 is of great interest. Herein, our results demonstrate that MMP-26 elevates the malignant phenotypes of MCF-7 breast cancer cells, including atypia, mitosis, spreading, migration and angiogenesis. Although the mechanisms underlying these effects have not been identified, these effects of MMP-26 have significant clinical implications. Since the high expression of MMP-26 is accompanied by increased malignant phenotypes, MMP-26 may be used as an important predictor in determining the degree of malignancy of breast cancer. Further studies are warranted to evaluate the role of MMP-26 in the prognosis of breast cancer.

\section{Acknowledgements}

This study was supported by the National Natural Science Foundation of China (grant nos. 30470662 and 30870970) and Jilin Provincial Science and Technology Projects (grant nos. 20050118, 200705358 and 20090513). We also acknowledge Dr F. William Orr for his critical suggestions on the organization of the manuscript.

\section{References}

1. Werb Z: ECM and cell surface proteopysis: regulating cellular ecology. Cell 91: 439-442, 1997.

2. Shin YJ and Kim JH: The role of EZH2 in the regulation of the activity of matrix metalloproteinases in prostate cancer cells. PLoS One 7: e30393, 2012.

3. Stetler-Stevenson WG: Matrix metalloproteinases in angiogenesis: a moving target for therapeutic intervention. J Clin Invest 103: 1237-1241, 1999.

4. Knopfova L, Benes P, Pekarcikova L, et al: c-Myb regulates matrix metalloproteinases $1 / 9$, and cathepsin D: implications for matrix-dependent breast cancer cell invasion and metastasis. Mol Cancer 11: 15, 2012.

5. Heymans S, Luttun A, Nuyens D, et al: Inhibition of plasminogen activators or matrix metalloproteinases prevents cardiac rupture but impairs therapeutic angiogenesis and causes cardiac failure. Nat Med 5: 1135-1142, 1999.

6. Ryggvason K, Höyhtyä M and Salo T: Proteolytic degradation of extracellular matrix in tumor invasion. Biochimica et Biophysica Acta 907: 191-217, 1987

7. Giannelli G, Falk-Marzillier J, Schiraldi O, et al: Induction of cell migration by matrix metalloprotease-2 cleavage of laminin-5. Science 277: 225-228, 1997.

8. Barnhill RL, Piepkorn MW, Cochran AJ, et al: Tumor vascularity, proliferation, and apoptosis in human melanoma micrometastases and macrometastases. Arch Dermatol 134: 991-994, 1998.

9. Park HI, Ni J, Gerkema FE, et al: Identification and characterization of human endometase (Matrix metalloproteinase-26) from endometrial tumor. J Biol Chem 275: 20540-20544, 2000.

10. Marchenko GN, Ratnikov BI, Rozanov DV, et al: Characterization of matrix metalloproteinase-26, a novel metalloproteinase widely expressed in cancer cells of epithelial origin. Biochem J 356: 705-718, 2001.

11. Marchenko ND, Marchenko GN, Weinreb RN, et al: Beta-catenin regulates the gene of MMP-26, a novel metalloproteinase expressed both in carcinomas and normal epithelial cells. Int $\mathrm{J}$ Biochem Cell Biol 36: 942-956, 2004.

12. Li W, Savinov AY, Rozanov DV, et al: Matrix metalloproteinase-26 is associated with estrogen-dependent malignancies and targets alpha1-antitrypsin serpin. Cancer Res 64: 8657-8665, 2004.

13. Zhang Y, Zhao H, Wang Y, Lin Y, Tan Y, Fang X and Zheng L: Non-small cell lung cancer invasion and metastasis promoted by MMP-26. Mol Med Report 4: 1201-1209, 2011.

14. Golubkov VS, Chekanov AV, Savinov AY, et al: Membrane type-1 matrix metalloproteinase confers aneuploidy and tumorigenicity on mammary epithelial cells. Cancer Res 66: 10460-10465,2006.

15. Golubkov VS and Strongin AY: Proteolysis-driven oncogenesis. Cell Cycle 6: 147-150, 2007.

16. Zhao YG, Xiao AZ, Newcomer RG, et al: Activation of pro-gelatinase B by endometase/matrilysin-2 promotes invasion of human prostate cancer cells. J Biol Chem 278: 15056-15064, 2003. 